\title{
Electron cloud buildup and related instability in the CERN Proton Synchrotron
}

\author{
R. Cappi, M. Giovannozzi, E. Métral, G. Métral, G. Rumolo, and F. Zimmermann \\ CERN, CH 1211 Geneva 23, Switzerland \\ (Received 14 May 2002; published 24 September 2002)
}

\begin{abstract}
The beam-induced electron cloud buildup is one of the major concerns for the SPS and the design of the future LHC. During the 2000 run, this effect has also been observed in the PS with the nominal LHC-type beam. The electron cloud induces a baseline distortion in electrostatic pickup signals, both during the last turns in the PS, when the full bunch length is reduced to less than $4 \mathrm{~ns}$, and in the transfer line between the PS and the SPS rings. In the year 2001, modifications in the rf hardware allowed us to study the properties of the beam instability related with the electron cloud phenomenon for a total bunch length of about $10 \mathrm{~ns}$. The complete set of experimental observations carried out in the PS machine is presented and discussed in detail.
\end{abstract}

DOI: 10.1103/PhysRevSTAB.5.094401

PACS numbers: 29.27.Bd, 29.27.Fh, 29.20.Lq

\section{INTRODUCTION}

Since the first studies concerning the potential harmful effect of the electron cloud buildup in the CERN Large Hadron Collider (LHC) machine (see [1], and references therein), the research shifted from purely theoretical to more experimental activities. After the Proton Synchrotron (PS) complex ${ }^{1}$ started to produce and deliver an LHC-like proton beam [3,4], the Super Proton Synchrotron (SPS) machine observed strong electron cloud buildup associated with a vertical instability $[5,6]$. Intense efforts were devoted to improve the understanding of the complex phenomena by using the SPS as a LHC test bed.

When the nominal LHC beam was generated by the PS machine [7], it was somewhat natural to investigate whether such a machine was also affected by electron cloud phenomena. It turned out that this was the case [8] and the standard signature, baseline drift in electrostatic devices, was observed. In fact, this should not be surprising as both machines work with the same type of beam and their vacuum chambers are quite similar: both are stainless steel pipes (the secondary emission yield is about 1.9) with comparable sizes (140 mm width, $70 \mathrm{~mm}$ height for PS and 129, $48.5 \mathrm{~mm}$ for SPS, respectively). Furthermore, the electron cloud buildup has also been observed in SPS regions with larger vertical half aperture, up to $70 \mathrm{~mm}$, thus making the similarity with the PS environment even stronger.

Thanks to the clean experimental conditions available in the PS machine, with a stable beam circulating on the high-energy flattop, further studies were devoted to the analysis of a possible electron cloud instability affecting the LHC beam. Interestingly, it turned out that, due to the very principle used to generate such a beam, the instability could not develop.

\footnotetext{
${ }^{1}$ For a review of the different types of beams normally delivered by the PS complex, see Ref. [2].
}

The nominal LHC beam at the exit of the PS consists of a train of 72 bunches, each of $1.1 \times 10^{11}$ protons, spaced by $25 \mathrm{~ns}$ and with a momentum of $26 \mathrm{GeV} / c$. The longitudinal emittance at $2 \sigma$ is $0.35 \mathrm{eVs}$ (obtained by means of successive longitudinal bunch splitting $[9,10])$, and the normalized rms transverse ones are $2.5 \mu \mathrm{m}$. Just before extraction, the bunches are compressed from $\sim 16$ to $\sim 4$ ns total length, within about 100 turns (i.e., about $200 \mu \mathrm{s})$. This is achieved by bunch rotation after a nonadiabatic increase of the rf voltage. The rotating bunches in the mismatched bucket are ejected after one quarter of the synchrotron period, when the minimum length is reached. A collection of the main parameters of the LHC beam and PS ring is listed in Table I.

However, by using adiabatic rf gymnastics it was only possible to reduce the total bunch length down to $10 \mathrm{~ns}$ and then to keep the bunches circulating for about $100 \mathrm{~ms}$.

TABLE I. Main parameters of the LHC beam and PS ring.

\begin{tabular}{lc}
\hline \hline Number of bunches & 72 \\
Bunch spacing $\left(T_{\text {sep }}\right)$ & $25 \mathrm{~ns}$ \\
Bunch population $\left(N_{b}\right)$ & $1.1 \times 10^{11}$ protons \\
Transverse rms emittances $\left(\epsilon_{x, y}^{*}\right)$ & $2.5 / 2.5 \mu \mathrm{m}$ \\
Chamber half aperture $(x)$ & $70 \mathrm{~mm}$ \\
Chamber half aperture $(y)$ & $35 \mathrm{~mm}$ \\
Tunes $\left(Q_{x, y, s}\right)$ & $6.25 / 6.25 / 0.0015$ \\
Bunch total length $\left(4 \sigma_{z} / c\right)$ & $4 \mathrm{~ns}$ \\
rms-momentum spread & $7 \times 10^{-4}$ \\
Longitudinal $2 \sigma$ emittance $\left(\epsilon_{l}\right)$ & $0.35 \mathrm{eVs}$ \\
Circumference & $628 \mathrm{~m}$ \\
Dipole field $\left(B_{y 0}\right)$ & $1.256 \mathrm{~T}$ \\
Field gradient $(G)$ & $5.2 \mathrm{~T} / \mathrm{m}$ \\
Relativistic $\gamma$ & 27.7 \\
$T_{\text {rev }}$ & $2.2 \mu \mathrm{s}$ \\
Average beta functions $\left(\beta_{x, y}\right)$ & $16 / 16 \mathrm{~m}$ \\
Average dispersion $\left(D_{x}\right)$ & $2.56 \mathrm{~m}$ \\
Momentum compaction $(\alpha)$ & 0.027 \\
Chromaticities $\left(\xi_{x, y}\right)$ & up to $0.5 \mathrm{in} \mathrm{both} \mathrm{planes}$ \\
\hline \hline
\end{tabular}

(C) 2002 The American Physical Society

094401-1 
Under these conditions, the beam instability was observed.

In the present paper, the observations made with the LHC-type beam in the PS ring and in the transfer line joining the PS to the SPS are described in Sec. II. In Sec. III the influence on the electron cloud buildup of the longitudinal bunch train structure and solenoidal field is presented. The outcome of the investigations concerning the beam instability is reported in Sec. IV, where also some possible remedies are outlined. Finally, conclusions are drawn in Sec. V.

\section{ELECTRON CLOUD BUILDUP}

\section{A. PS ring}

An electron cloud buildup was observed during the last turns in the PS, when the bunch compression takes place. The electron cloud induces baseline distortions in electrostatic pickup signals. The effect is essentially visible in the vertical plane, as illustrated in Fig. 1. The pickup has the bandwidth $0.2-30 \mathrm{MHz}$ and is located near a combined function bending magnet. These distortions are not present when either the bunch rotation is not active or other beams (i.e., non-LHC-type) are observed.

\section{B. TT2 transfer line between PS and SPS}

The electron cloud buildup was also observed in the TT2 (single-pass) transfer line between PS and SPS (see Fig. 2). Here the pickup is located in a field-free region, and its bandwidth is $0.006-400 \mathrm{MHz}$. The capacitance of the device is $C=500 \mathrm{pF}$ and the voltage corresponding to the drift of the baseline is estimated to be $V \approx$ $300 \mathrm{mV}$. Hence the number of electrons pulled out of the electrostatic pickup electrodes is given by

$$
n_{e}=\frac{C V}{e} \approx 10^{9}
$$

However, in this case electron cloud effects seem to lead only to instrumentation problems: observations indicate that the beam quality, i.e., the beam position and the transverse beam emittance, are not affected. It will be shown later that this is due to the fact that the time of electron cloud-beam interaction is short compared to the rise time of the related instability. All these phenomena are a peculiarity of the nominal LHC beam, i.e., no distortion is visible when observing other beams delivered by the PS.

Finally, it is worthwhile mentioning that electron cloud buildup also perturbs the emittance measurement devices installed in the TT2 transfer line. The standard approach to determine the beam emittance, provided the dispersion function is known all along a transfer line, consists of measuring transverse beam profiles at three different locations [11]. The devices installed in TT2 are secondary emission monitors made of thin wires. In Fig. 3 (left panels) typical horizontal beam profiles for the LHCtype beam without the final bunch rotation are shown. All of them have a nice Gaussian-like shape. However, when the bunch rotation is turned on, beam profiles are strongly perturbed (see the right panels of Fig. 3).

A clear nonzero signal is present even when the monitors are retracted from the beam, provided the bunch rotation is active. Of course, no signal is present in the absence of bunch rotation (no electron cloud buildup) and with the monitors out of beam. No disturbance of the beam profiles is present with bunch rotation but larger bunch spacing (e.g., 50 ns) or with a reduced bunch intensity.

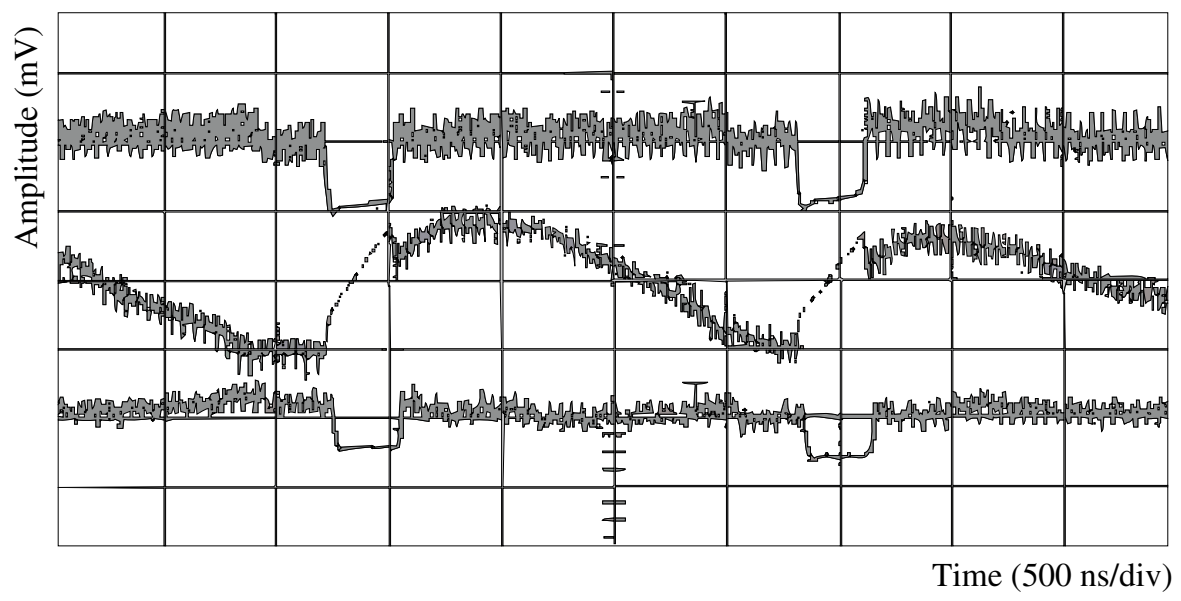

FIG. 1. Measured baseline drift in a PS electrostatic pickup during bunch compression prior to extraction. From top to bottom: $\Sigma(300 \mathrm{mV} / \mathrm{div}), \Delta y(20 \mathrm{mV} / \mathrm{div})$, and $\Delta x(200 \mathrm{mV} / \mathrm{div})$. The $30 \mathrm{MHz}$ bandwidth of the pickup does not allow discriminating the $4 \mathrm{~ns}$ long bunches. The bunch train lasts $1.8 \mu$ s and the gap is $320 \mathrm{~ns}$. 

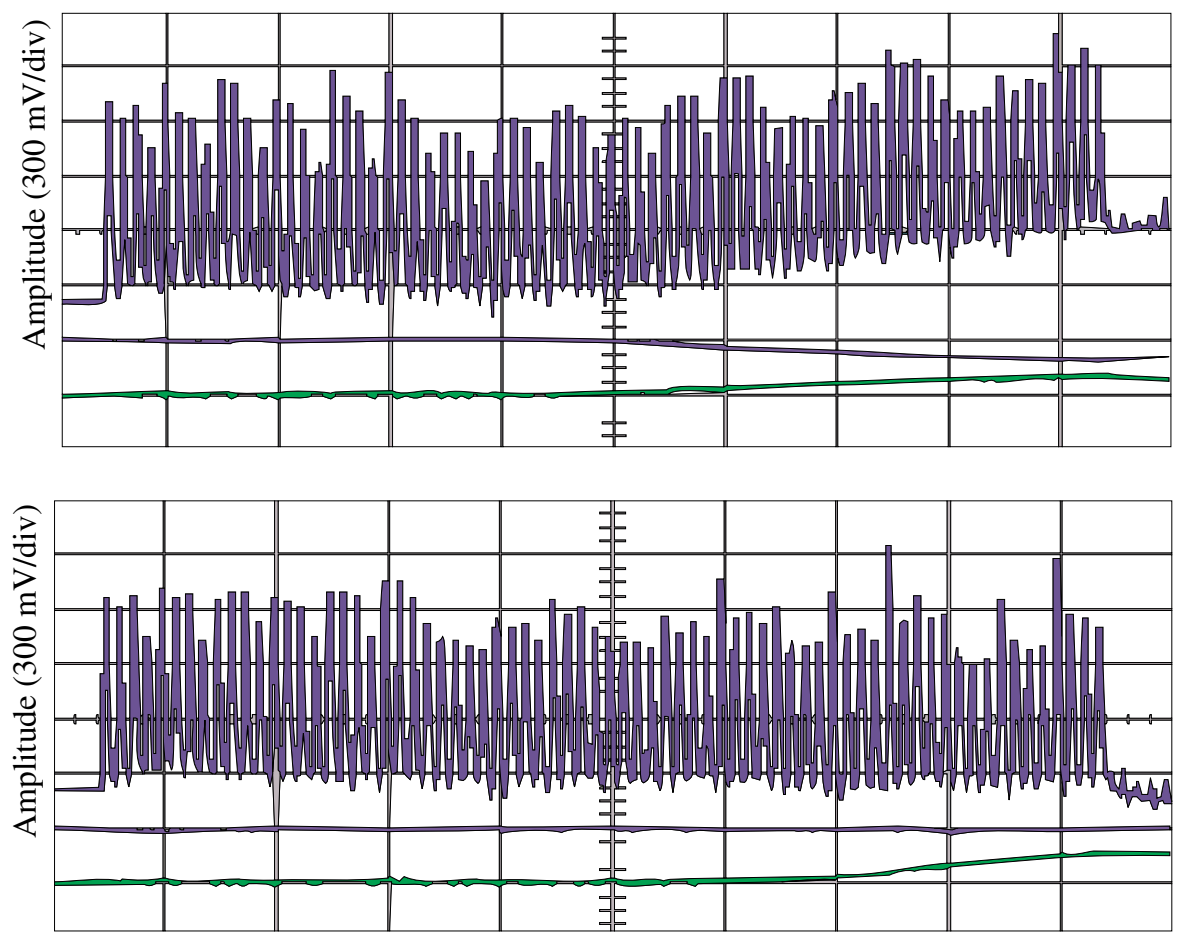

Time (200 ns/div)

FIG. 2. (Color) Measured baseline drift in a TT2 electrostatic pickup: $I_{\text {solenoid }}=0$ (upper panel) and $I_{\text {solenoid }}=10 \mathrm{~A}$ (lower panel). The details of the solenoid will be discussed in Sec. III C. From top to bottom: $\Sigma, \Delta x$, and $\Delta y(\Delta x, \Delta y$ signals almost coincide with the grid lines). This pickup has a bandwidth wide enough to allow discriminating the short bunches. Such a structure is not observable on $\Delta x$ and $\Delta y$ signals as the beam is centered in the pickup.
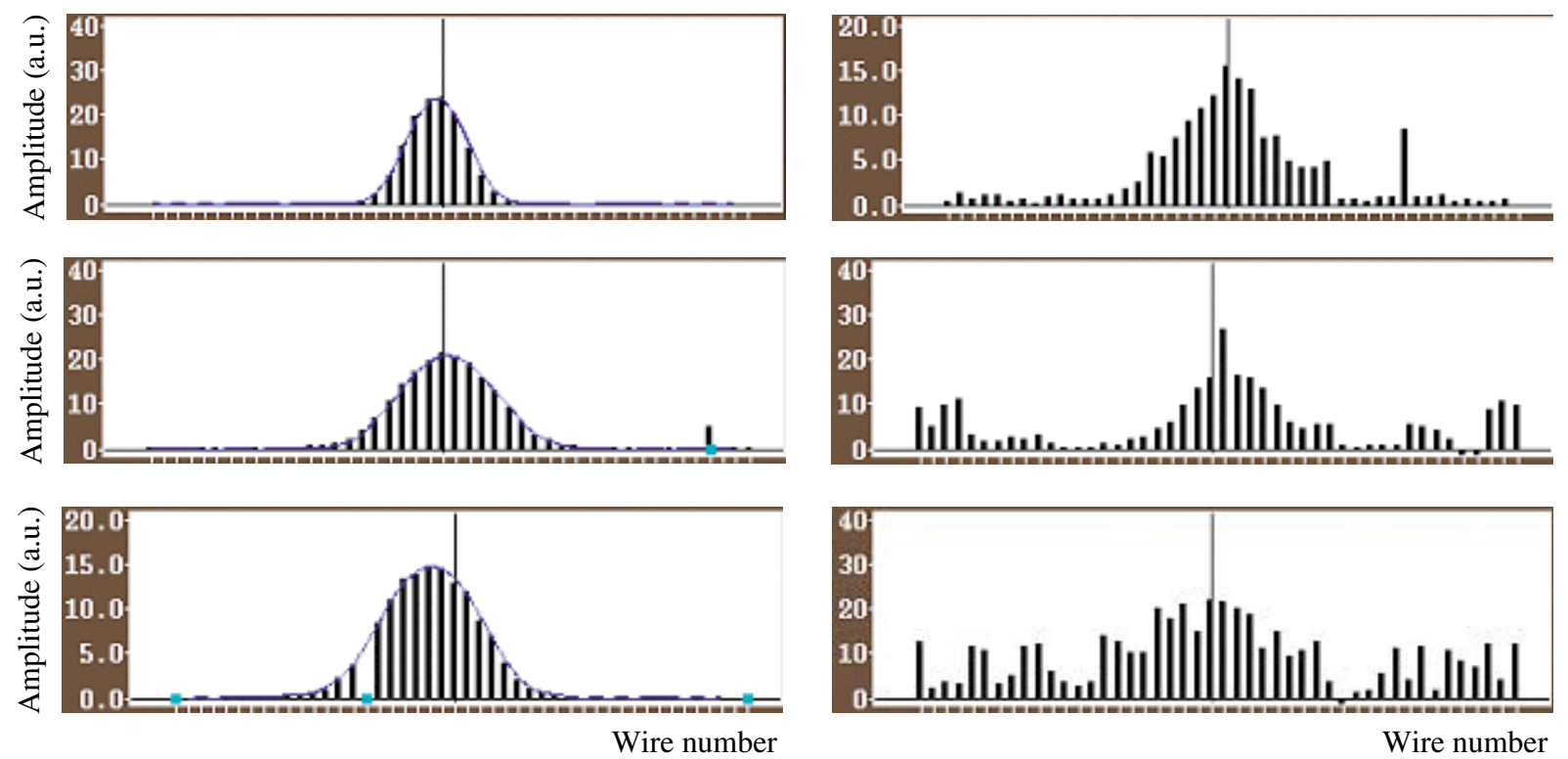

FIG. 3. (Color) Transverse horizontal beam profiles measured with secondary emission monitors at three different locations in the TT2 transfer line. The measurements performed without bunch rotation are shown on the left, while the profiles on the right are obtained with the final bunch rotation active. The wire spacing is $0.5 \mathrm{~mm}$ for all the monitors except the central one where the spacing is $0.35 \mathrm{~mm}$. 


\section{CONTROL OF ELECTRON CLOUD BUILDUP}

The buildup of an electron cloud can be either suppressed or strongly reduced by acting on a number of physical parameters. Observations were made of the dependence on the bunch spacing and on the presence of gaps in the bunch train. Furthermore, the effect of a solenoidal field on the dynamics of the electron cloud was also studied.

\section{A. Bunch spacing}

A variant of the nominal LHC beam with a larger bunch spacing, $50 \mathrm{~ns}$ instead of $25 \mathrm{~ns}$, was tested during the years 2000 and 2001. Originally, the presence of strong longitudinal coupled bunch instabilities made it impossible to achieve the nominal intensity per bunch. After a series of improvements on the high order mode of the rf cavities [12], it was possible to obtain the nominal beam intensity, namely, $N_{b} \approx 1.1 \times 10^{11}$ protons per bunch $(\mathrm{p} / \mathrm{b})$, during the 2001 run.

The signals detected on a pickup in the PS machine (upper panel) and in the TT2 transfer line (lower panel) are shown in Fig. 4. Under these conditions the electron cloud buildup is substantially reduced: not only is the baseline drift in the $\Sigma$ signal smaller than for the $25 \mathrm{~ns}$ case, but it also starts later along the bunch train.

\section{B. Gaps in the bunch train}

The evolution of the baseline drift in a TT2 electrostatic pickup is depicted in Fig. 5, where gaps of 12 bunches (corresponding to $320 \mathrm{~ns}$ ) are introduced. The gap is obtained by removing one PS Booster bunch at PS injection. Electron cloud buildup always shows up at the end of the bunch train, independently of the gap position. These measurements show that a gap of $320 \mathrm{~ns}$ is not sufficient to reset the memory of the electron cloud: the electron cloud density is rapidly reestablished behind the gap.

Observations performed in the presence of six gaps of $120 \mathrm{~ns}$ are shown in Fig. 6. Even in this case a drift of the baseline is visible at the end of the bunch train.

Finally, the case of 84 bunches filling 84 buckets at harmonic number $h=84$ was also studied. The signals are shown in Fig. 7, where the measurements made with a pickup in the last turns in the PS machine (upper panel) and in the TT2 transfer line (lower panel) are shown.

The buildup of the electron cloud is the strongest among the different cases considered here. Yet, even
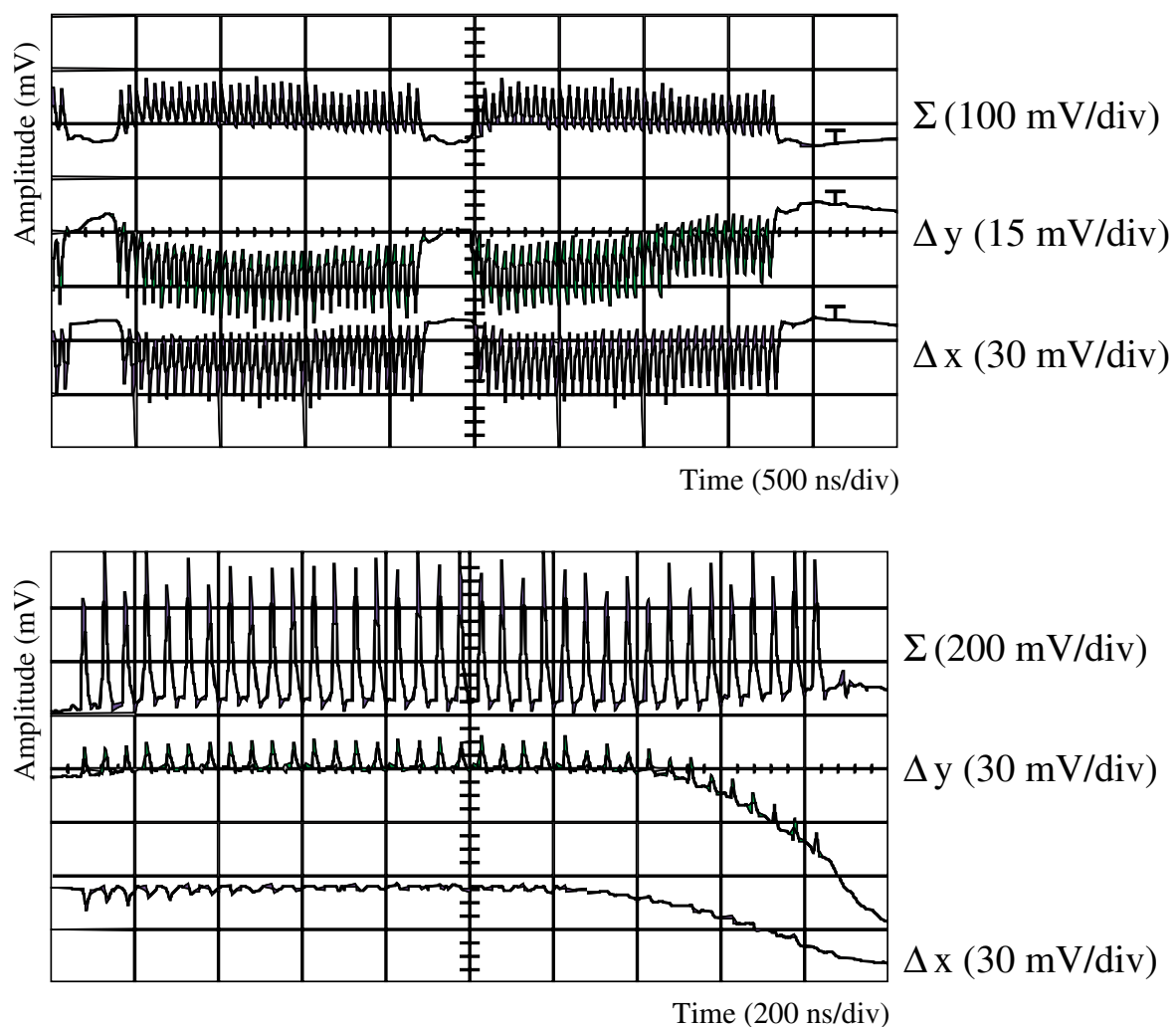

FIG. 4. Measured baseline drift in a PS electrostatic pickup (upper panel) and in TT2 (lower panel) for the $50 \mathrm{~ns}$ spacing LHC-type beam. The electron cloud buildup is delayed with respect to the $25 \mathrm{~ns}$ case and the drift is also reduced. 

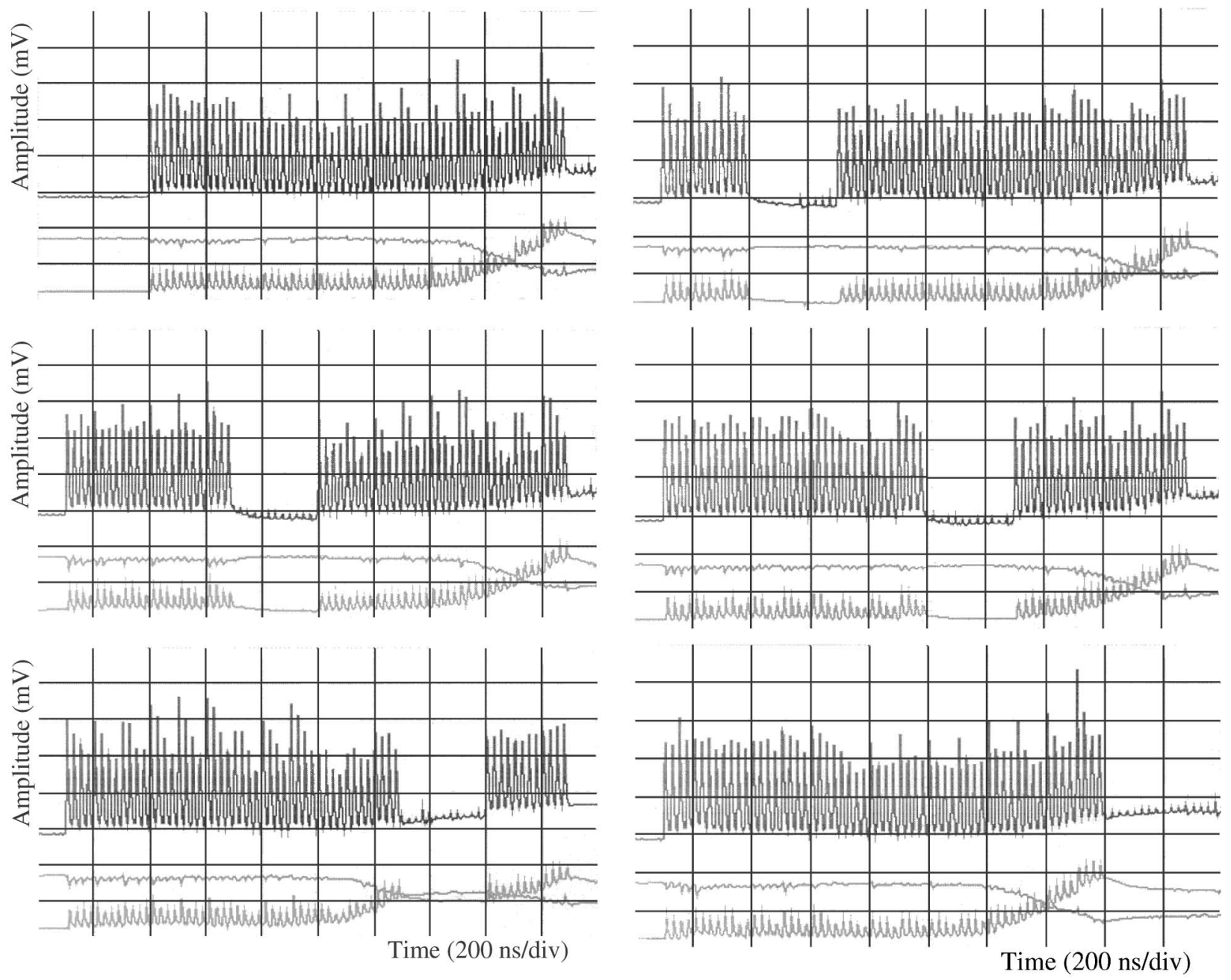

FIG. 5. Measured baseline drift in a TT2 electrostatic pickup, with 6 possible cases of 12 missing bunches. From top to bottom: $\Sigma$ (800 mV/div), $\Delta y(100 \mathrm{mV} /$ div $)$, and $\Delta x(100 \mathrm{mV} /$ div $)$.

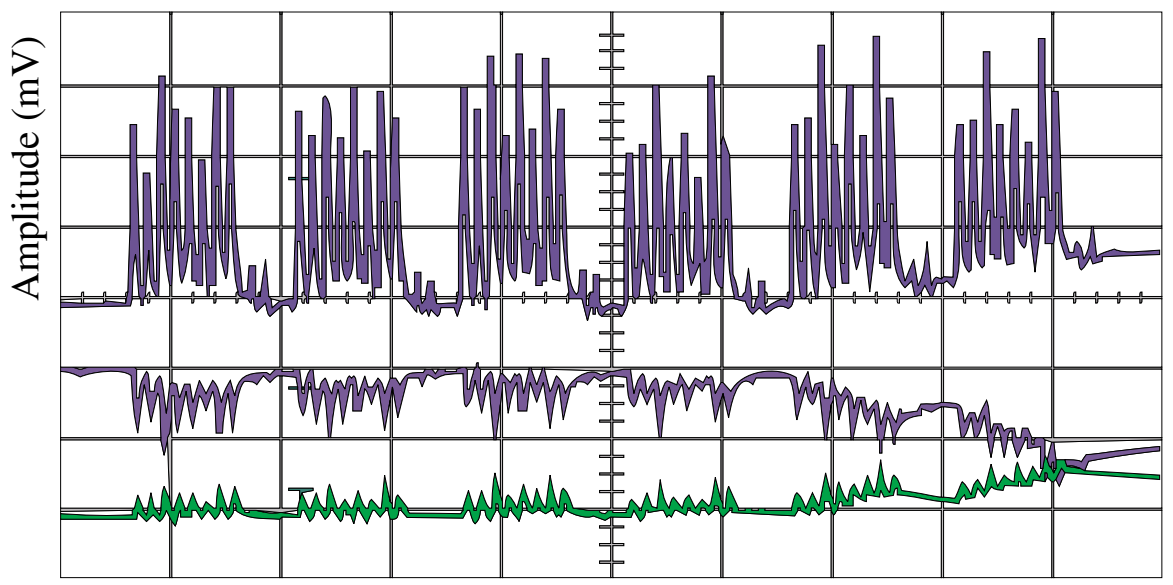

Time (200 ns/div)

FIG. 6. (Color) Measured baseline drift in a TT2 electrostatic pickup with six gaps of $120 \mathrm{~ns}$. From top to bottom: $\Sigma(300 \mathrm{mV} / \mathrm{div}), \Delta x(50 \mathrm{mV} / \mathrm{div})$, and $\Delta y(50 \mathrm{mV} / \mathrm{div})$. 

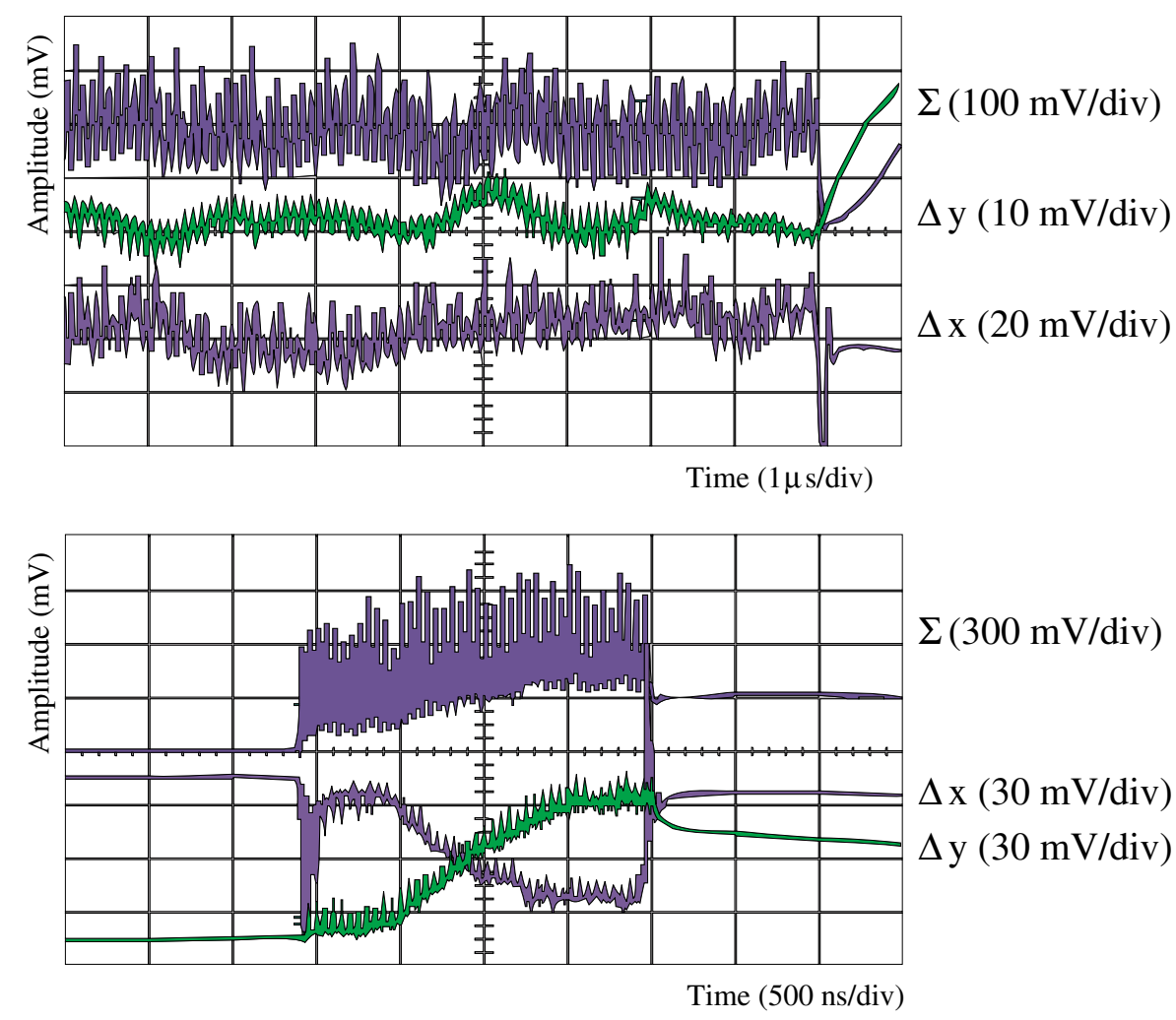

FIG. 7. (Color) Measured baseline drift in a PS electrostatic pickup in the last turns before extraction (upper panel) and in TT2 (lower panel) for the 25 ns spacing with 84 bunches (i.e., no gap). A strong electron cloud buildup is clearly visible. The spike on the $\Delta x$ signal for the TT2 pickup is generated by the bunches badly ejected due to the finite rise time of the extraction kicker.

under these extreme conditions, which were obtained for only a fraction of a millisecond, the beam was stable.

\section{Solenoidal field}

The investigations related to the electron cloud buildup in the B-factories and lepton rings, such as KEKB, PEPII, and BEPC, clearly showed the beneficial effect of the solenoidal field on the machine performance (see Refs. [13-15]). In fact, the longitudinal solenoid field, although quite small, keeps the electrons close to the chamber wall and thus suppresses the beam-induced multipacting.

Hence, the same technique was applied in the TT2 transfer line to confirm the hypothesis that the source of the perturbations of the beam diagnostics was really the electron cloud buildup. To this end, coils were installed at both ends of the wide-band electrostatic pickup. Each of the coils is made of 80 windings over a length of about $0.08 \mathrm{~m}$. The coils have an inner diameter of about $0.13 \mathrm{~m}$ and an outer one of about $0.25 \mathrm{~m}$. The distance between the two extreme ends of the coils is $0.4 \mathrm{~m}$, while the longitudinal size of the pickup is about $0.16 \mathrm{~m}$. The maximum current is $10 \mathrm{~A}$. The pickup, together with the additional coils used to generate the solenoidal field can be seen in Fig. 8.

The value of the longitudinal component of the magnetic field along the axis of the pickup is shown in Fig. 9.

By applying a weak solenoidal field in the TT2 electrostatic pickup, the baseline distortion could be eliminated (see Fig. 2). A residual effect is visible, however, on the vertical signal, which may be due to the nonideal solenoidal field created.

\section{INSTABILITIES}

\section{A. Experimental observations}

After having detected the electron cloud buildup, the next step consisted in studying the related instability in more detail.

It was already clear that for the nominal LHC beam the electron cloud only perturbed the beam diagnostics, the beam quality being unaffected. Hence, a different approach was used where the method of producing the beam was modified. Instead of applying a nonadiabatic 


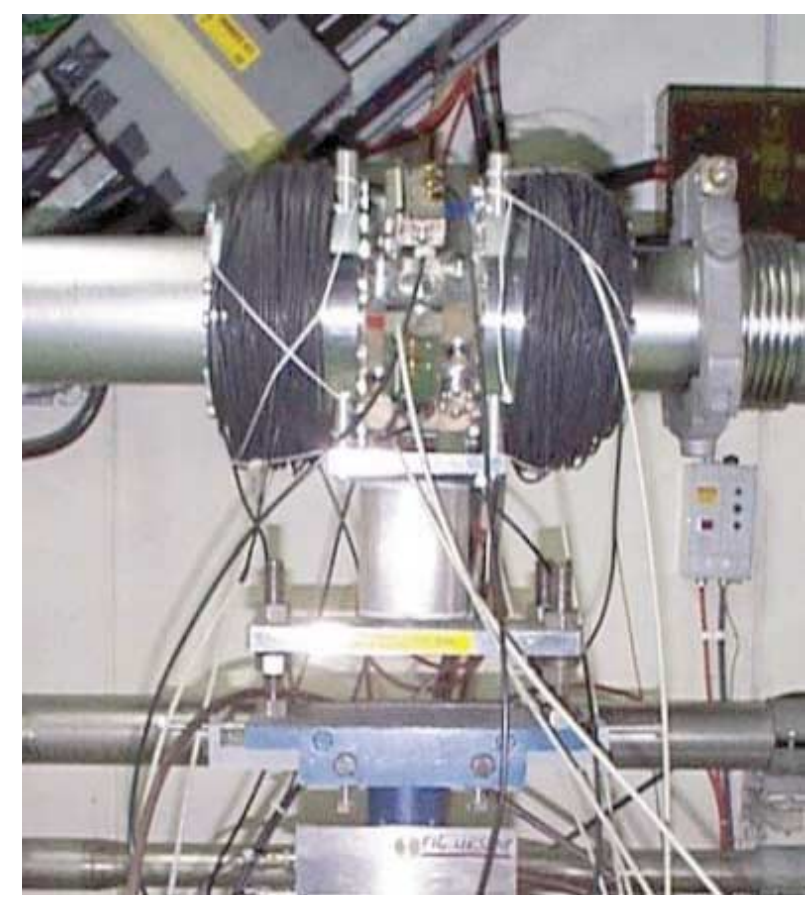

FIG. 8. (Color) View of the electrostatic pickup installed in the TT2 transfer line. The two coils are clearly seen on both sides of the device.

bunch rotation by using $80 \mathrm{MHz}$ cavities on top of the $40 \mathrm{MHz}$, an adiabatic rf gymnastics was used.

The signal of a horizontal pickup in the PS ring was frequency analyzed to keep track of the evolution of the first unstable betatron line (about $357 \mathrm{kHz}$ ). It was possible to observe a horizontal single-bunch instability with a threshold at $N_{b}^{t h} \approx 4.6 \times 10^{10} \mathrm{p} / \mathrm{b}$ and a rise time of $3-$ $4 \mathrm{~ms}$ above the threshold. In Fig. 10 such a signal is shown for six different values of $N_{b}$. All the plots cover a time interval of $200 \mathrm{~ms}$ before beam extraction. The first case refers to an intensity just below the threshold, while the

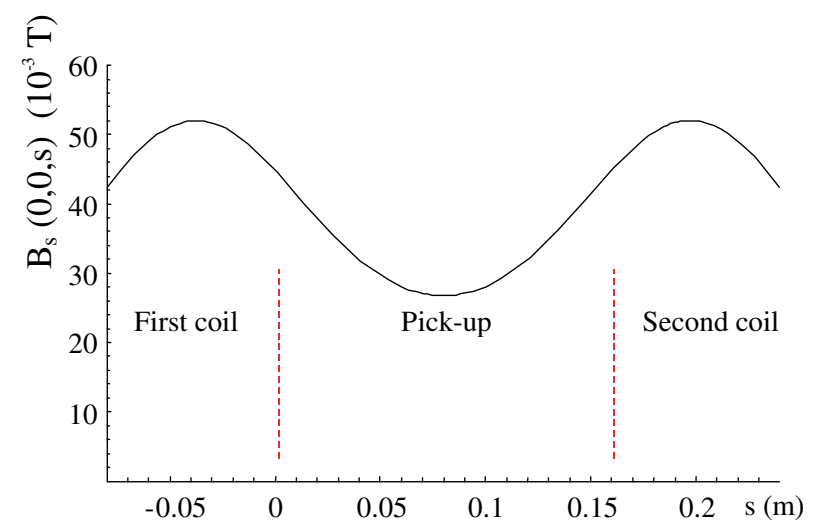

FIG. 9. (Color) Absolute value of the longitudinal component of the magnetic field $B_{s}(0,0, s)$ generated by the two coils installed at both ends of the wide-band electrostatic pickup for the maximum current. second one is just above it. The rise time is higher than in the other cases, but this is due to the fact that the measurement is taken near the threshold. The linear amplitude increase in the logarithmic scale is clearly visible in five out of the six cases plotted in Fig. 10.

It is clearly seen how the instability rise time is almost constant above the threshold. However, the instability appears earlier in time. During the $200 \mathrm{~ms}$ shown in the plots the beam undergoes a double-splitting gymnastics (lasting about $55 \mathrm{~ms}$ ), then it stays with a bunch length of $16 \mathrm{~ns}$ for about $40 \mathrm{~ms}$ before being adiabatically compressed, reaching a bunch length of about $10 \mathrm{~ns}$ in $5 \mathrm{~ms}$. Finally, the bunches are in equilibrium during the last $100 \mathrm{~ms}$ before extraction. Therefore, it seems that for the highest intensities the electron cloud buildup occurs already for bunches longer than $10 \mathrm{~ns}$ : in fact, the multipacting regime is originated by the interplay of both bunch intensity and bunch length.

Some additional information for the case $N_{b} \approx 5.5 \times$ $10^{10} \mathrm{p} / \mathrm{b}$ is shown in Fig. 11. Together with the evolution of the first unstable betatron line (upper left panel), the Fourier analysis of the same signal is shown in the upper right panel. In the lower part, the signals from pickups in the PS ring (left panel) and TT2 transfer line (right panel) are shown.

It is clear that (i) the strong instability is visible only in the horizontal plane, and (ii) no regular pattern can be detected in the horizontal position along the bunch train. This seems to rule out a multibunch instability.

Two points should be stressed: first, the PS lattice is made of combined function magnets (dipole field with quadrupole component); second, the fraction of machine circumference occupied by the combined function elements is about $70 \%$. Therefore, it is clear that the characteristics of the beam instability will be dictated by the properties of the electron cloud in the main magnets. In addition, due to the peculiar field configuration of a combined function magnet, the property that the wake field in the horizontal plane is close to zero, as for a vertical dipole field, no longer holds true [16], which may explain why a horizontal instability can develop.

\section{B. Remedies}

Before going into more details in the analysis of the remedies to be applied to combat the electron cloud driven instability, it is necessary to stress that two different phenomena have to be distinguished: the electron cloud buildup and the instability. Electron cloud buildup from beam-induced multipacting occurs only if the bunch intensity is high enough so as to accelerate electrons to energies of about $100 \mathrm{eV}$, near the maximum of the secondary emission yield. As far as the instability is concerned, it can occur only if the electron cloud is produced. Therefore, one could conclude that all the remedies discussed in previous sections to prevent the 

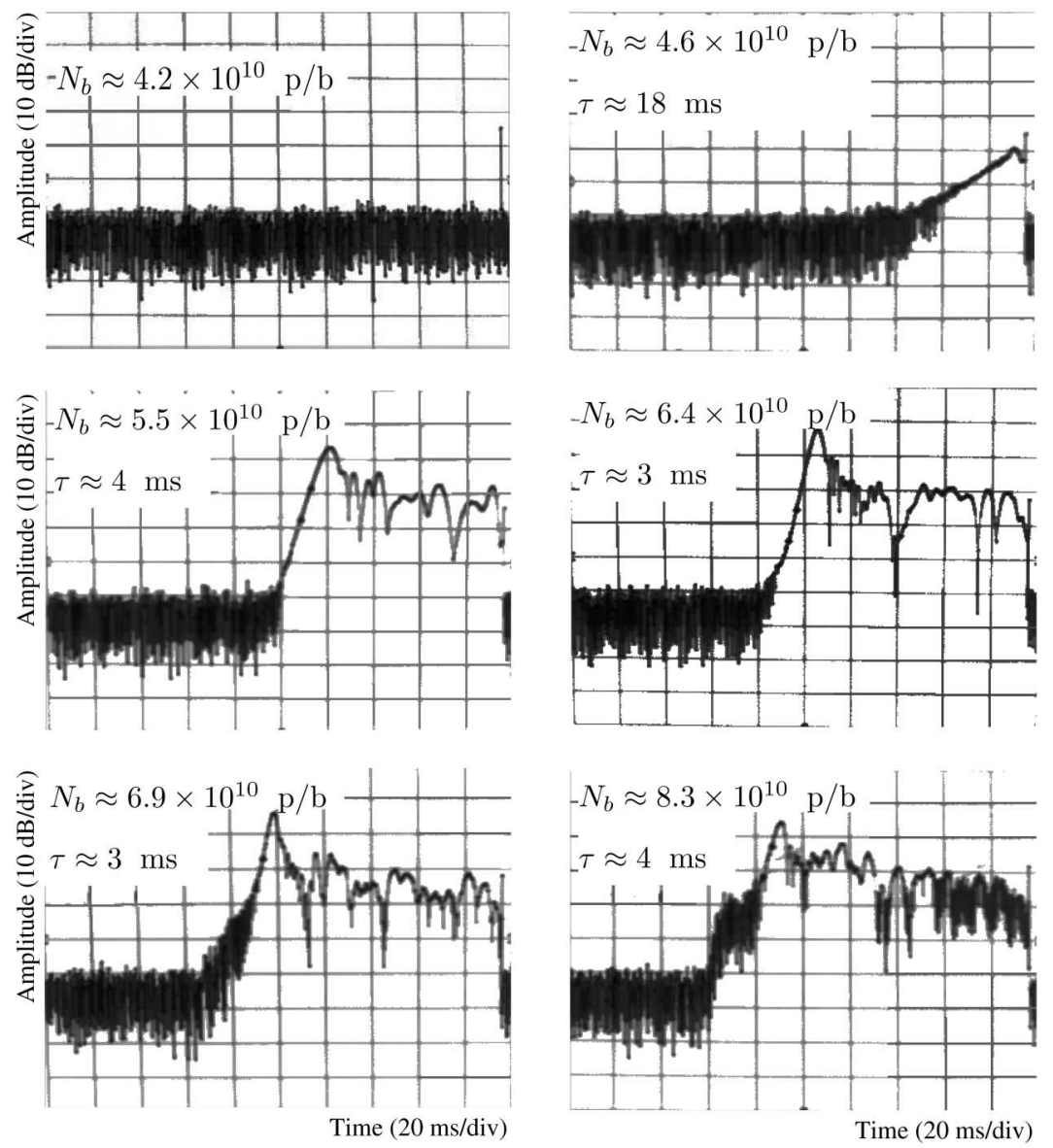

FIG. 10. Rise time of the horizontal instability as a function of the bunch intensity. The signal is obtained with a spectrum analyzer with zero frequency span and a central frequency $357 \mathrm{kHz}$. The beam is extracted at the end of the horizontal scale.
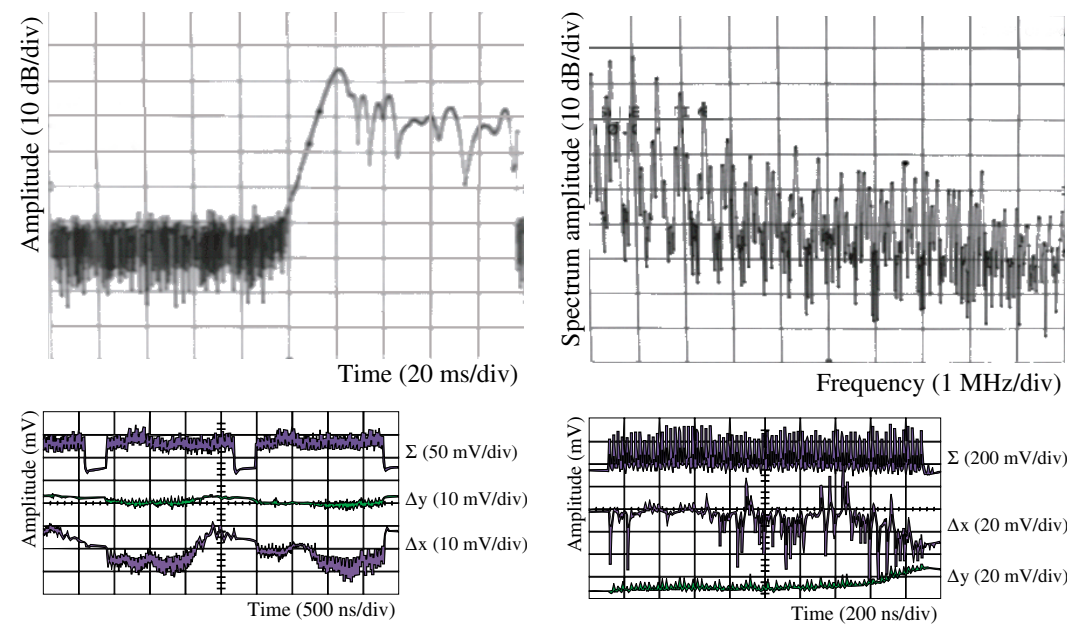

FIG. 11. (Color) Instability footprint for $N_{b} \approx 5.5 \times 10^{10} \mathrm{p} / \mathrm{b}$. The signal obtained via a spectrum analyzer with zero frequency span and central frequency of $357 \mathrm{kHz}$ is shown in the upper left panel. A Fourier analysis from $0-10 \mathrm{MHz}$ is shown in the upper right panel. In the lower part, the signals from a pickup in the PS ring some tens of milliseconds before extraction (left panel) and the TT2 transfer line (right panel) are shown. 


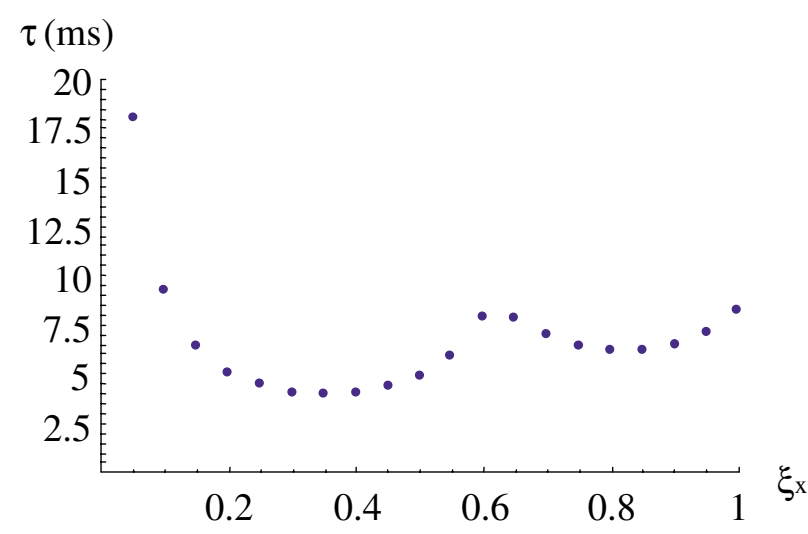

FIG. 12. (Color) Dependence of the horizontal head-tail instability rise time $\tau$ on the horizontal chromaticity $\xi_{x}$ for the theoretical model of the PS.

electron cloud buildup are, in a certain sense, also remedies for the instability. However, here we will discuss the techniques that can be applied to stabilize the beam once the cloud is generated and the beam becomes unstable.

As a first attempt, the horizontal chromaticity $\xi_{x}$, defined as

$$
\xi_{x}=\frac{\Delta Q_{x}}{Q_{x}} / \frac{\Delta p}{p_{0}},
$$

where $Q_{x}$ is the horizontal betatronic tune, $p$ the particle's momentum, and $p_{0}$ the reference particle's momentum, has been changed. Normally it is set to about 0.1 on the high-energy flattop. However, similarly to what was done in the SPS [5], $\xi_{x}$ was increased up to $\xi_{x} \approx 0.5$, but no variation in the instability rise time was detected.

This fact is in good agreement with theoretical estimates [17] obtained by approximating the electron cloud with a broadband impedance [18] and using the amplitude of the horizontal wake field computed for the PS main magnet [16]. The resulting instability rise time $\tau$ as a function of $\xi_{x}$ is shown in Fig. 12: the weak dependence of $\tau$ on $\xi_{x}$ in the range $0.15 \leq \xi_{x} \leq 0.5$ is clearly seen.

Finally, octupoles were powered in an attempt to stabilize the beam. Under these new conditions, only a marginal effect was observed, despite the rather large current used (almost near to the maximum sustainable by the power converter). The corresponding octupoleinduced tune spread at half width half height $[19,20]$ can be estimated to be $\Delta Q_{x, \mathrm{HWHH}} \approx 4 \times 10^{-5}$ and $\Delta Q_{y, \mathrm{HWHH}} \approx 5 \times 10^{-5}$.

\section{CONCLUSIONS AND OUTLOOK}

Since generating the required LHC beam in the PS, intense experimental efforts were devoted to measuring electron cloud buildup and related instabilities. As far as the nominal beam is concerned, the conditions to generate an electron cloud buildup are met only when no time is left for the related instability to develop. This is due to the specific rf manipulation, a nonadiabatic bunch rotation performed a few turns before beam extraction.

However, it was possible to study the buildup both at extraction in the PS ring and in the transfer line between the PS and SPS, as a function of some bunch parameters such as bunch spacing, gaps in the bunch train, and presence of a solenoidal field around an electrostatic pickup.

These observations lead to the conclusion that, for the nominal LHC beam, the electron cloud buildup does not alter the beam characteristics. Such a cloud only constitutes a perturbing effect for the different types of beam diagnostics, such as electrostatic pickups and secondary emission monitors.

By introducing a modified rf gymnastic (an adiabatic bunch rotation reducing the bunch length from 16 to $10 \mathrm{~ns}$ ), it was possible to keep the shortened beam circulating for about $100 \mathrm{~ms}$. Under these conditions, it was possible to observe a horizontal single-bunch instability with threshold $N_{b}^{\text {th }} \approx 4.6 \times 10^{10} \mathrm{p} / \mathrm{b}$, and rise time $\tau$ of about 3-4 ms above threshold. No sign of instability was observed in the vertical plane. This seems to be linked to the peculiarity of the PS lattice whose main magnets are combined function magnets. According to preliminary numerical simulations, the main wake field is produced in the horizontal plane. The observations could be explained by the head-tail formalism.

The influence of chromaticity was measured, revealing only a marginal effect on the beam dynamics, in agreement with theoretical predictions. Finally, the effect of octupoles was also tried out, showing only a small stabilizing effect. It is worth mentioning that a variant of the nominal LHC beam with a $50 \mathrm{~ns}$ bunch spacing completely removed the instability.

Additional measurements are foreseen for the year 2002 run, to get more quantitative results on the properties of the beam instability related to the electron cloud buildup.

\section{ACKNOWLEDGMENTS}

We would like to thank the PS operation crew and the experts of the rf and beam diagnostics groups for constant support during the measurement campaign.

[1] F. Zimmermann, CERN LHC Project Report No. 95, 1997 (unpublished).

[2] D. J. Simon, for the PS Staff, in Proceedings of the Fifth European Particle Accelerator Conference, Barcelona, 1996, edited by C. Petit-Jean-Genaz et al. (Institute Of Physics, Bristol, 1996), p. 295.

[3] R. Cappi, R. Garoby, M. Martini, J.-P. Riunaud, and K. Schindl, CERN Report No. PS 94-11 (DI), 1994 (unpublished). 
[4] K. Schindl, for the PS Staff, in Proceedings of the Fourth European Particle Accelerator Conference, London, 1994, edited by V. Suller et al. (Edition Frontièrs, Gif sur Yvette, 1994), p. 500.

[5] G. Arduini et al., in Proceedings of the Seventh European Particle Accelerator Conference, Vienna, 2000, edited by J. Poole and Ch. Petit-Jean-Genaz (European Physical Society, Geneva, 2000), p. 939.

[6] G. Arduini et al., in Proceedings of the Seventh European Particle Accelerator Conference, Vienna, 2000 (Ref. [5]), p. 259.

[7] M. Benedikt, R. Cappi, M. Chanel, R. Garoby, M. Giovannozzi, S. Hancock, M. Martini, E. Métral, G. Métral, K. Schindl, and J.-L. Vallet, CERN Report No. PS 2001-011 (DR), 2001 (unpublished).

[8] R. Cappi, M. Giovannozzi, E. Métral, G. Métral, and F. Zimmermann, in Proceedings of the 2001 Particle Accelerator Conference, edited by P.W. Lucas and S. Webber (IEEE, Piscataway, NJ, 2001), p. 685.

[9] R. Garoby, in Proceedings of the Seventeenth International High-Energy Accelerators Conference HEACC's98, edited by I. N. Meshkov (Joint Institute Nuclear Research, Dubna, 1998), p. 172.

[10] R. Garoby, S. Hancock, and J.-L. Vallet, in Proceedings of the Seventh European Particle Accelerator Conference, Vienna, 2000 (Ref. [5]), p. 304.

[11] P. J. Bryant and K. Johnsen, Circular Accelerators and Storage Rings (Cambridge University Press, New York, 1993).
[12] R. Garoby, in Proceedings of LHC Workshop Chamonix XI, Chamonix, 2001, edited by J. Poole (unpublished).

[13] Y. Funakoshi et al., in Proceedings of the Seventh European Particle Accelerator Conference, Vienna, 2000 (Ref. [5]), p. 28.

[14] J.T. Seeman et al., in Proceedings of the Seventh European Particle Accelerator Conference, Vienna, 2000 (Ref. [5]), p. 38.

[15] Z.Y. Guo et al., in Proceedings of the 2001 Particle Accelerator Conference (Ref. [8]), p. 676.

[16] M. Giovannozzi, E. Métral, G. Métral, G. Rumolo, and F. Zimmermann, in Proceedings of the Mini-Workshop on Electron-Cloud Simulations for Proton and Positron Beams, CERN Geneva, 2002, edited by G. Rumolo and F. Zimmermann (unpublished).

[17] E. Métral, in Proceedings of the Mini-Workshop on Electron-Cloud Simulations for Proton and Positron Beams, CERN Geneva, 2002 (Ref. [16]) (unpublished).

[18] K. Ohmi, E. Perevedentsev, and F. Zimmermann, CERN Report No. SL 2001-011 (AP), 2001 (unpublished).

[19] D. Möhl, CERN Report No. PS 95-08 (DI), 1995 (unpublished).

[20] R. Cappi, R. Garoby, and E. Métral, in Proceedings of the Workshop on Instabilities of High Intensity Hadron Beams in Rings, edited by T. Roser and S. Y. Zhang (American Institute of Physics, New York, 1999), p. 116. 